This item was submitted to Loughborough's Research Repository by the author.

Items in Figshare are protected by copyright, with all rights reserved, unless otherwise indicated.

\title{
Guided surface waves on one- and two-dimensional arrays of spheres
}

PLEASE CITE THE PUBLISHED VERSION

http://dx.doi.org/10.1137/100787519

\section{PUBLISHER}

(c) Society for Industrial and Applied Mathematics (SIAM)

\section{VERSION}

VoR (Version of Record)

\section{LICENCE}

CC BY-NC-ND 4.0

\section{REPOSITORY RECORD}

Thompson, lan, and C.M. Linton. 2019. "Guided Surface Waves on One- and Two-dimensional Arrays of Spheres". figshare. https://hdl.handle.net/2134/15304. 
This item was submitted to Loughborough's Institutional Repository (https://dspace.lboro.ac.uk/) by the author and is made available under the following Creative Commons Licence conditions.

\section{creative
commons}

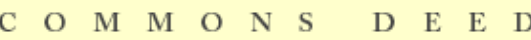

Attribution-NonCommercial-NoDerivs 2.5

You are free:

- to copy, distribute, display, and perform the work

Under the following conditions:

Attribution. You must attribute the work in the manner specified b the author or licensor.

Noncommercial. You may not use this work for commercial purposes.

No Derivative Works. You may not alter, transform, or build upon this work.

- For any reuse or distribution, you must make clear to others the license terms of this work.

- Any of these conditions can be waived if you get permission from the copyright holder.

Your fair use and other rights are in no way affected by the above.

This is a human-readable summary of the Leqal Code (the full license).

\section{Disclaimer 만}

For the full text of this licence, please go to: http://creativecommons.org/licenses/by-nc-nd/2.5/ 


\title{
GUIDED SURFACE WAVES ON ONE- AND TWO-DIMENSIONAL ARRAYS OF SPHERES*
}

\author{
I. THOMPSON $^{\dagger}$ AND C. M. LINTON $^{\dagger}$
}

\begin{abstract}
Guided acoustic waves propagating along one- and two-dimensional arrays of rigid spheres are studied semianalytically. The quasi-periodic wavefield is constructed as a superposition of spherical wave functions, and then application of the boundary condition on the sphere surfaces leads to an infinite system of real linear algebraic equations. The vanishing of the determinant of the associated infinite matrix provides the condition for surface waves to exist, and these are determined numerically. In the case of a two-dimensional array, we consider arbitrary skew lattices and compute surface modes which are either symmetric or antisymmetric about the plane of the array. Our numerical calculations make extensive use of previous work by the authors on the accurate and efficient computation of lattice sums.
\end{abstract}

Key words. arrays, spheres, surface waves

AMS subject classifications. 74J20, 74J15, 78A45

DOI. $10.1137 / 100787519$

1. Introduction. Wave interaction with infinite periodic arrays of spheres has been studied extensively in a variety of physical settings. It is important to draw a distinction between three-dimensional arrays which fill the whole of space and oneand two-dimensional arrays which allow for wave propagation towards or away from the spheres. It is these latter cases that we are concerned with here.

Acoustic scattering by a two-dimensional array of spheres was first considered by Twersky [42, 40,41], though no numerical results were presented in these papers. The equivalent electromagnetic problem has also been considered in the context of low energy electron diffraction [27, 34]. A theory for acoustic wave scattering by an infinite linear array of identical obstacles was also developed by Twersky [39]. Twersky's approach to multiple scattering (described for the two-dimensional case in [23, section 6.13]) is ingenious, but it does not lead to equations which are suitable for numerical computation. Instead, Twersky focused on deriving analytic properties of the solutions and approximate solutions for small scatterers or low frequencies, and these in turn have been used to help validate other approximate solutions; see, e.g., [4]. It should be noted that Twersky's method applies to obstacles of quite general shape, though there is considerable simplification if the scatterers are spherically symmetric. Scattering of a longitudinal elastic wave by a doubly periodic array of spherical inclusions in an infinite elastic solid was treated in [1] using a boundary integral equation method (and used subsequently [2] as a building block in the computation of dispersion curves for the propagation of waves in an elastic solid containing a three-dimensional array of regularly spaced spherical cavities). The reflection and transmission of a longitudinal plane wave by a distribution of spherical cavities situated in the interface between two solids with different mechanical properties was investigated, again using boundary integral equations, in [44]. A full vector formalism based on multipoles for treating elastic scattering by periodic arrays of spherical scatterers was given in [22]

\footnotetext{
* Received by the editors March 3, 2010; accepted for publication (in revised form) July 15, 2010; published electronically October 5, 2010.

http://www.siam.org/journals/siap/70-8/78751.html

${ }^{\dagger}$ Department of Mathematical Sciences, Loughborough University, Leicestershire, LE11 3TU, UK (i.thompson@lboro.ac.uk, c.m.linton@lboro.ac.uk).
} 
and used to study scattering by a planar sheet of spheres, a problem also treated in $[30,31]$. Some experimental results on the diffraction of ultrasonic waves by a periodic array of spherical inclusions in an elastic matrix are presented in $[14,24,25]$.

In this article we consider both linear and two-dimensional arrays of spheres, but rather than study how an incident wave is scattered by the array, we are interested in the ability of the array to support guided wave modes that travel along the array (in the absence of an incident field) and decay exponentially as one moves away from the array. In fact the formulations for these two types of problem are very similar, and much of our analysis could easily be modified for use in scattering problems. Guided modes propagating along periodic structures have been studied extensively in a number of different physical contexts in two dimensions and are known variously as edge waves [8], Rayleigh-Bloch surface waves [28], array-guided surface waves [12], and bound states [43]. These waves are distinct from guided waves which propagate along surfaces introduced into photonic or phononic crystals, which have also been studied in recent years (for example, in electromagnetism [7] and in elasticity theory [37]). There have been previous studies of electromagnetic surface waves guided by periodic arrays, but these have focused on situations where the spheres can be modeled by some combination of electric and magnetic dipoles [32, 3, 16, 33].

The particular physical problem that we focus on is sound waves in the presence of rigid spheres. We actually formulate the problem for penetrable spheres as this does not increase the complexity of the problem in any significant way, but for the purposes of numerical results we focus on the special case of Neumann boundary conditions so as to keep the number of free parameters manageable. Our goal is to provide a thorough study of the modes that exist in this case, both for the problem's intrinsic interest and as a first step towards the more challenging electromagnetic problem, which will be the subject of a subsequent article. Our problem is a natural extension of the equivalent two-dimensional problem involving cylinders [26] and, as in that case, there is a cut-off frequency below which waves cannot radiate energy away from the array. The modes that we seek have frequencies below this cut-off. We do not address the far more difficult question of whether surface modes exist at frequencies above the cut-off (in which case they are usually referred to as embedded modes); a review of work on embedded modes in two dimensions can be found in [19].

In section 2 we formulate the general problem of acoustic scattering by a single penetrable sphere, and in section 3 we use this to derive the equations appropriate to an infinite one- or two-dimensional array. The specific case of a linear array is treated in section 4 , with numerical results presented in section 5 , while the two-dimensional array and associated numerical results are given in section 6 and section 7 , respectively. All of the wavefields considered in this article are time-harmonic, and a factor $\mathrm{e}^{-\mathrm{i} \omega t}$ is assumed throughout. In addition, we will use the conventions that $|\mathbf{v}|=v$ and $\hat{\mathbf{v}}=\mathbf{v} / v$ for any vector $\mathbf{v}$.

2. Acoustic wave interactions with spheres. We begin by considering a single sphere of radius $a$ centered at the origin; later this is used as a building block for constructing modes in the presence of multiple spheres. In the region exterior to the sphere, i.e., where $r>a$, the wavefield $u(\mathbf{r})$ must satisfy the Helmholtz equation

$$
\left(\nabla^{2}+k^{2}\right) u(\mathbf{r})=0
$$

where $k=\omega / c$, with $c$ representing the speed of sound. In this section, circumflexed symbols are used to refer to quantities inside the sphere. Thus, in the interior region $r<a$, the wavefield is represented by $\hat{u}(\mathbf{r})$, and this must also satisfy the Helmholtz 
equation, but with $k$ replaced by $\hat{k}=\omega / \hat{c}$. General expansions for wavefields outside and inside the sphere can be derived by separation of variables. For $r \geq a$, we have

$$
u(\mathbf{r})=\sum_{n, m}\left(C_{n}^{m} \mathcal{J}_{n}^{m}(\mathbf{r})+A_{n}^{m} \mathcal{H}_{n}^{m}(\mathbf{r})\right)
$$

whereas for $r \leq a$,

$$
\hat{u}(\mathbf{r})=\sum_{n, m} \hat{A}_{n}^{m} \mathcal{J}_{n}^{m}(\mathbf{r} \hat{k} / k)
$$

The regular and outgoing spherical wave functions $\mathcal{J}$ and $\mathcal{H}$ are defined in (A.4). Note that the field inside the sphere does not involve outgoing wave functions; these are singular at $r=0$. Also, in view of (A.1), we have $\mathcal{H}_{n}^{m}(\mathbf{r}) \equiv \mathcal{J}_{n}^{m}(\mathbf{r}) \equiv 0$ for $|m|>n$, and so we have introduced the shorthand notation

$$
\sum_{n, m} \equiv \sum_{n=0}^{\infty} \sum_{m=-n}^{n}
$$

which will be used throughout. The first and second terms in (2.2) represent the incident field and scattered response, respectively. Thus in a scattering problem the coefficients $C_{n}^{m}$ are known at the outset, whereas $A_{n}^{m}$ and $\hat{A}_{n}^{m}$ are to be determined. The boundary conditions, representing continuity of pressure and normal velocity, are

$$
u=\hat{u} \quad \text { and } \quad \frac{1}{\rho} \frac{\partial u}{\partial r}=\frac{1}{\hat{\rho}} \frac{\partial \hat{u}}{\partial r} \quad \text { on } \quad r=a,
$$

in which $\rho$ and $\hat{\rho}$ are the densities of the exterior and interior media, respectively. Applying these, and exploiting the orthogonality property (A.8), we eliminate $\hat{A}_{n}^{m}$ to obtain

$$
A_{n}^{m}+Z_{n} C_{n}^{m}=0
$$

where the scattering coefficient $Z_{n}$ is given by

$$
Z_{n}=\frac{q \mathrm{j}_{n}^{\prime}(k a) \mathrm{j}_{n}(\hat{k} a)-\mathrm{j}_{n}(k a) \mathrm{j}_{n}^{\prime}(\hat{k} a)}{q \mathrm{~h}_{n}^{\prime}(k a) \mathrm{j}_{n}(\hat{k} a)-\mathrm{h}_{n}(k a) \mathrm{j}_{n}^{\prime}(\hat{k} a)} .
$$

Here, $\mathrm{j}_{n}(\cdot)$ and $\mathrm{h}_{n}(\cdot)$ represent spherical Bessel and spherical Hankel functions of the first kind, respectively, and $q=\hat{\rho} \hat{c} / \rho c$. Note that setting $q=0$ is equivalent to imposing Dirichlet (sound-soft) boundary conditions $(u=0$ on $r=a)$, whereas letting $q \rightarrow \infty$ recovers the Neumann (rigid) case $(\partial u / \partial r=0$ on $r=a)$. Equation (2.6) allows us to construct the scattered response without reference to the expansion of the interior field (2.3). The scattering coefficients have the special property that the quantity $W_{n}$, defined as

$$
W_{n}=\frac{\mathrm{i} Z_{n}}{1-Z_{n}}=\frac{q \mathrm{j}_{n}^{\prime}(k a) \mathrm{j}_{n}(\hat{k} a)-\mathrm{j}_{n}(k a) \mathrm{j}_{n}^{\prime}(\hat{k} a)}{q \mathrm{y}_{n}^{\prime}(k a) \mathrm{j}_{n}(\hat{k} a)-\mathrm{y}_{n}(k a) \mathrm{j}_{n}^{\prime}(\hat{k} a)},
$$

is real, the importance of which will become apparent later. 
3. Guided waves on $\boldsymbol{d}$-dimensional arrays of spheres. In general, a lattice of dimension $d$ is the set of points with position vectors

$$
\mathbf{R}_{n}=\sum_{i=1}^{d} n_{i} \mathbf{s}_{i}, \quad n_{i} \in \mathbb{Z},
$$

where $n=\left(n_{1}, \ldots, n_{d}\right)$ is a multi-index. The set of all lattice vectors $\mathbf{R}_{n}$ is denoted by $\Lambda$. In cases where $d>1$, the basis vectors $\mathbf{s}_{i}$ are not necessarily orthogonal (though they are linearly independent) and need not have the same length. We will be concerned here only with the cases $d=1$ and $d=2$. The reciprocal lattice consists of the points with position vectors

$$
\mathbf{R}_{n}^{*}=2 \pi \sum_{i=1}^{d} n_{i} \mathbf{s}_{i}^{*}, \quad n_{i} \in \mathbb{Z},
$$

where $\mathbf{s}_{i} \cdot \mathbf{s}_{j}^{*}=\delta_{i j}$, and the set of all reciprocal lattice vectors $\mathbf{R}_{m}^{*}$ is denoted by $\Lambda^{*}$. Crucially, for any lattice vector $\mathbf{R}_{n}$ and any reciprocal lattice vector $\mathbf{R}_{m}^{*}$,

$$
\mathrm{e}^{\mathrm{i} \mathbf{R}_{n} \cdot \mathbf{R}_{m}^{*}}=1
$$

The Poisson summation formula for a $d$-dimensional lattice, which will be required later, is given by

$$
\sum_{\mathbf{R}_{n} \in \Lambda} \mathrm{e}^{-\mathrm{i} \mathbf{K} \cdot \mathbf{R}_{n}}=\frac{(2 \pi)^{d}}{\mathcal{A}} \sum_{\mathbf{R}_{n}^{*} \in \Lambda^{*}} \delta\left(\mathbf{K}-\mathbf{R}_{n}^{*}\right),
$$

where $\mathcal{A}=s_{1}$ when $d=1$, and $\mathcal{A}=\left|\mathbf{s}_{1} \times \mathbf{s}_{2}\right|$ (i.e., the area of a lattice cell) when $d=2$.

An array is constructed by centering identical spheres of radius $a$ at each lattice point. According to Bloch's theorem [13, pp. 32-35], a guided wave that propagates along such a structure satisfies the quasi-periodicity condition

$$
u\left(\mathbf{r}+\mathbf{R}_{j}\right)=\mathrm{e}^{\mathrm{i} \boldsymbol{\beta} \cdot \mathbf{R}_{j}}(\mathbf{r}), \quad \mathbf{R}_{j} \in \Lambda,
$$

for some $d$-dimensional Bloch vector $\boldsymbol{\beta}$. Thus, if we represent the mode as a sum of contributions emanating from each sphere, then we have

$$
u(\mathbf{r})=\sum_{\mathbf{R}_{j} \in \Lambda} \mathrm{e}^{\mathrm{i} \boldsymbol{\beta} \cdot \mathbf{R}_{j}} \sum_{n, m} A_{n}^{m} \mathcal{H}_{n}^{m}\left(\mathbf{r}_{j}\right),
$$

where $\mathbf{r}_{j}=\mathbf{r}-\mathbf{R}_{j}$ is a position vector relative to the center of sphere $j$. In order to be physically realizable, the mode (3.6) must not radiate energy away from the array into the far field. In fact it is sufficient to check that

$$
u(\mathbf{r}+\mathbf{v}) \rightarrow 0 \quad \text { as } \quad v \rightarrow \infty \quad \text { for any } \mathbf{v} \perp \mathbf{s}_{i}, i=1, \ldots, d,
$$

since it will subsequently be shown that this actually implies exponential decay.

The complex coefficients $A_{n}^{m}$ are determined by applying the boundary conditions on the surface of the sphere centered at the origin. Boundary conditions elsewhere then follow by quasi-periodicity. To this end, we write

$$
u(\mathbf{r})=\sum_{n, m} A_{n}^{m} \mathcal{H}_{n}^{m}(\mathbf{r})+\sum_{n, m} A_{n}^{m} \sum_{\mathbf{R}_{j} \in \Lambda}^{\prime} \mathrm{e}^{\mathrm{i} \boldsymbol{\beta} \cdot \mathbf{R}_{j}} \mathcal{H}_{n}^{m}\left(\mathbf{r}_{j}\right)
$$


where the prime indicates that the term with $R_{j}=0$ is to be omitted from the summation. The first term on the right-hand side is the field emanating from the central sphere, whereas the second is the field incident on that sphere, which consists of contributions radiating from all of the other spheres. This latter term can be expanded about $r=0$ as a series of regular wave functions using the addition theorem (A.13). In this way, we obtain an expression of the form (2.2), with

$$
C_{n}^{m}=\sum_{\nu, \mu} A_{\nu}^{\mu} \sum_{\mathbf{R}_{j} \in \Lambda}^{\prime} \mathrm{e}^{\mathrm{i} \boldsymbol{\beta} \cdot \mathbf{R}_{j}} S_{\nu n}^{\mu m}\left(-\mathbf{R}_{j}\right)
$$

where the separation matrix $\left[S_{\nu n}^{\mu m}\right]$ is given by (A.14). This expansion is valid, provided that $r<R_{j}$ for all nonzero $\mathbf{R}_{j} \in \Lambda$. Applying the boundary conditions using (2.6) now leads to the homogeneous linear system of equations

$$
A_{n}^{m}+Z_{n} \sum_{\mathbf{R}_{j} \in \Lambda}^{\prime} \mathrm{e}^{\mathrm{i} \boldsymbol{\beta} \cdot \mathbf{R}_{j}} \sum_{\nu, \mu} S_{\nu n}^{\mu m}\left(-\mathbf{R}_{j}\right) A_{\nu}^{\mu}=0, \quad n \geq|m| .
$$

The existence of guided waves along the array corresponds to values of $k$ and $\boldsymbol{\beta}$ for which (3.10) admits nontrivial solutions for the unknowns $A_{n}^{m}$ and for which the radiation condition (3.7) is also satisfied. In fact, each such set of parameters reveals a second solution that represents a wave traveling in the opposite direction. This second solution can be obtained directly from the first by replacing $\mathbf{R}_{j}, \boldsymbol{\beta}$, and $A_{n}^{m}$ with $-\mathbf{R}_{j}$, $-\boldsymbol{\beta}$, and $(-1)^{n} A_{n}^{m}$, respectively, and applying the symmetry relation (A.16) (note that $\left.\mathbf{R}_{j} \in \Lambda \Leftrightarrow-\mathbf{R}_{j} \in \Lambda\right)$.

4. Linear array. In the one-dimensional case we may dispense with the subscripts for basis vectors (since there is now only one of these) and so $s=|\mathbf{s}|$ is the spacing between the lattice points. Clearly we must have $2 a \leq s$ so that the spheres do not overlap. The line of sphere centers is chosen as the axis of a spherical coordinate system $(r, \theta, \phi)$ so that $z=r \cos \theta$. If we denote the unit vector in the $z$-direction by $\mathbf{e}_{z}$, we then have $\mathbf{s}=s \mathbf{e}_{z}, \mathbf{R}_{j}=j s \mathbf{e}_{z}$, and $\boldsymbol{\beta}=\tilde{\beta} \mathbf{e}_{z}$ (see Figure 1). In view of (3.5), we may assume without loss of generality that

$$
-\pi / s<\tilde{\beta} \leq \pi / s .
$$

In fact, we need only consider $\tilde{\beta} \geq 0$, due to the symmetry of the system in the plane $z=0$ (see section 3 above). A great deal of simplification now occurs in the linear system for the coefficients $A_{n}^{m}$ due to the axisymmetric nature of the geometry. Thus,

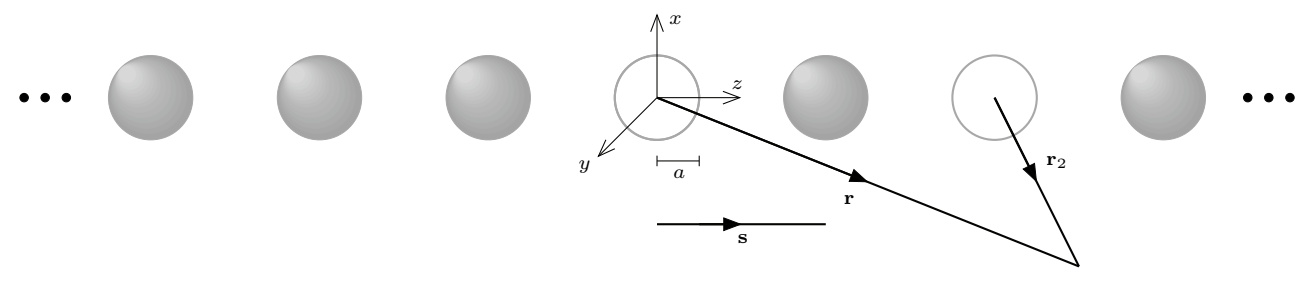

FIG. 1. Schematic diagram showing a section of the linear array and the coordinate system in use. The sphere centers are at $\mathbf{r}=\mathbf{R}_{j}=j \mathbf{s}$, and a position vector relative to sphere $j$ is defined via $\mathbf{r}_{j}=\mathbf{r}-\mathbf{R}_{j}$. 
on writing the separation matrix explicitly from (A.17), (3.10) becomes

$$
A_{n}^{m}+4 \pi Z_{n} \sum_{\nu=|m|}^{\infty} A_{\nu}^{m} \sum_{\substack{q=|n-\nu| \\ n+\nu-q \text { even }}}^{n+\nu} \mathrm{i}^{2 m+n-q-\nu} \mathcal{G}(\nu, m ; n,-m ; q) \sigma_{q}(\tilde{\beta})=0, \quad n \geq|m| .
$$

Here, $\mathcal{G}$ is a Gaunt coefficient (see the appendix), and we have defined the lattice sum

$$
\sigma_{q}(\tilde{\beta})=\lambda_{q 0} \sum_{j=1}^{\infty} \mathrm{h}_{q}(k j s)\left(\mathrm{e}^{\mathrm{i} j s \tilde{\beta}}+(-1)^{q} \mathrm{e}^{-\mathrm{i} j s \tilde{\beta}}\right),
$$

with $\lambda_{q 0}$ given by (A.6). Note that the azimuthal modes have decoupled, leading to a separate problem for each distinct value of $m$. Also, changing $m$ to $-m$ has no effect on (4.2) in view of (A.19), and so we need only seek solutions in cases where $m \geq 0$. Once a solution to (4.2) has been obtained, the field is given by (3.6) with a fixed value of $m$, that is,

$$
u^{m}(\mathbf{r})=\sum_{j=-\infty}^{\infty} \mathrm{e}^{\mathrm{i} j s \tilde{\beta}} \sum_{n=|m|}^{\infty} A_{n}^{m} \mathcal{H}_{n}^{m}\left(\mathbf{r}_{j}\right) .
$$

An alternative form for $u(\mathbf{r})$ can be obtained by inserting the integral representation (A.12) and then using the Poisson summation formula (3.4). We find that

$$
u^{m}(\mathbf{r})=\mathrm{e}^{\mathrm{i} m \phi} \sum_{j=-\infty}^{\infty} \mathcal{M}_{j}^{m} \mathrm{e}^{\mathrm{i} \tilde{\beta}_{j} z} \mathrm{~K}_{m}\left(k \sqrt{x^{2}+y^{2}} \gamma_{j}\right)
$$

where $\mathrm{K}_{m}(\cdot)$ is a modified Bessel function and the amplitude coefficient is given by

$$
\mathcal{M}_{j}^{m}=\frac{2 \mathrm{i}}{k s}(-1)^{m+1} \sum_{n=|m|}^{\infty} \mathrm{i}^{-n} A_{n}^{m} \lambda_{n m} \mathrm{P}_{n}^{m}\left(\tilde{\beta}_{j} / k\right)
$$

Here, $\lambda_{m n}$ and $P_{n}^{m}(\cdot)$ are defined in (A.6) and (A.1), respectively, and we have written

$$
\tilde{\beta}_{j}=\tilde{\beta}+2 j \pi / s \text { and } \gamma_{j}=\gamma\left(\tilde{\beta}_{j} / k\right),
$$

where the function $\gamma(\cdot)$ is defined in (A.11). Equation (4.5) is an expansion in terms of conical waves, and only those for which $\gamma_{j}$ is imaginary radiate energy away from the array. If there are no values of $j$ such that $\left|\tilde{\beta}_{j}\right| \leq k$, which happens when

$$
k<\tilde{\beta} \leq \pi / s,
$$

then $\gamma_{j}>0$ for all $j$ and the radiation condition (3.7) is satisfied. This is a sufficient but not necessary condition. Another possibility is that $\left|\tilde{\beta}_{j}\right| \leq k$ for one or more integers $j$ but that $\mathcal{M}_{j}^{m}=0$ for the same values, so that (3.7) is still satisfied. Examples of these so-called embedded modes have been constructed in related twodimensional problems $[10,29]$, but we are not concerned with such phenomena here. When (4.8) is satisfied, a final simplification of (4.2) occurs, because in this case [21]

$$
\mathrm{i}^{q} \sigma_{q}=\mathrm{i} \eta_{q}-\delta_{q 0} / \sqrt{4 \pi}
$$


where $\eta_{q}$ is real. After substituting (4.9) into (4.2), using (A.19), and then dividing the entire system by $1-Z_{n}$, we obtain

$$
\begin{aligned}
\left(\mathrm{i}^{-n} A_{n}^{m}\right)+4 \pi W_{n} \sum_{\nu=m}^{\infty}\left(\mathrm{i}^{-\nu} A_{\nu}^{m}\right) \sum_{\substack{q=|n-\nu| \\
n+\nu-q \text { even }}}^{n+\nu}(-1)^{m+q} \mathcal{G}(\nu, m ; n,-m ; q) \eta_{q}(\tilde{\beta})=0 \\
n \geq m \geq 0
\end{aligned}
$$

where $W_{n}$ is given by (2.8). This is a real homogeneous system of equations for the unknowns $\left(\mathrm{i}^{-n} A_{n}^{m}\right)$.

Now the lattice sum $\sigma_{q}$ (and therefore $\eta_{q}$ ) has logarithmic singularities at points where $\tilde{\beta}_{j}= \pm k$ for some $j \in \mathbb{Z}$. In particular, from [21], we have

$$
\eta_{q}(\tilde{\beta})=\widehat{\eta}_{q}(\tilde{\beta})+(-1)^{q} \frac{\lambda_{q 0}}{k s} \ln (\tilde{\beta} s-k s),
$$

where $\widehat{\eta}_{q}$ remains bounded as $\tilde{\beta} \rightarrow k$. The other singularities of $\eta_{q}$ are of no concern here in view of (4.8). By substituting (4.11) into (4.10), we can separate the terms that are singular in the limit $\tilde{\beta} \rightarrow k$ from those that are regular. In the former case, we can then evaluate the sum over $q$ using the linearization formula for spherical harmonics [23, Theorem 3.20]. We find that

$$
\begin{aligned}
& \left(\mathrm{i}^{-n} A_{n}^{m}\right)+4 \pi W_{n} \sum_{\nu=m}^{\infty}\left(\mathrm{i}^{-\nu} A_{\nu}^{m}\right)\left[a_{\substack{m \\
m}}^{m}+\sum_{\substack{q=|n-\nu| \\
n+\nu-q \text { even }}}^{n+\nu}(-1)^{m+q} \mathcal{G}(\nu, m ; n,-m ; q) \widehat{\eta}_{q}(\tilde{\beta})\right]=0, \\
& n \geq m,
\end{aligned}
$$

where

$$
a_{n \nu}^{m}=\frac{\delta_{m 0}}{k s} \lambda_{n 0} \lambda_{\nu 0} \ln (\tilde{\beta} s-k s) .
$$

Thus, in cases where $m \neq 0$, all of the terms in (4.12) are bounded for all values of $\tilde{\beta}$ and $k$ permitted by $(4.8)$.

5. Numerical results for one-dimensional arrays. Extensive numerical searches for guided waves with azimuthal mode numbers $m=0, \ldots, 10$ that can propagate along linear arrays were carried out using program code written in Fortran 2003. The infinite linear system (4.10) (which converges exponentially) was truncated at $n=\nu=N$, where $N$ is chosen so that $\left|W_{N+1}\right|<\epsilon\left|W_{0}\right|$, where $\epsilon \approx 10^{-15}$ represents machine precision. The Gaunt coefficients were computed using an updated version of the Root-Rational-Fraction Package described in [35], and the lattice sums were evaluated using the method in [21]. We present our results with the problem scaled so that the spacing between consecutive sphere centers $(s)$ is unity, and so $a \leq 0.5$.

No solutions were found in the case of Dirichlet boundary conditions (which is consistent with the fact that no such waves exist in the equivalent two-dimensional problem of waves propagating along circular arrays of cylinders [5]). In the case of Neumann boundary conditions, modes were found to exist, but only when $m=0$. Figure 2(a) shows the difference between the Bloch wavenumber $\tilde{\beta}$ and the free-space wavenumber $k$ for various sphere radii. Typically, $\tilde{\beta}-k$ increases with both $k$ and $a$, 

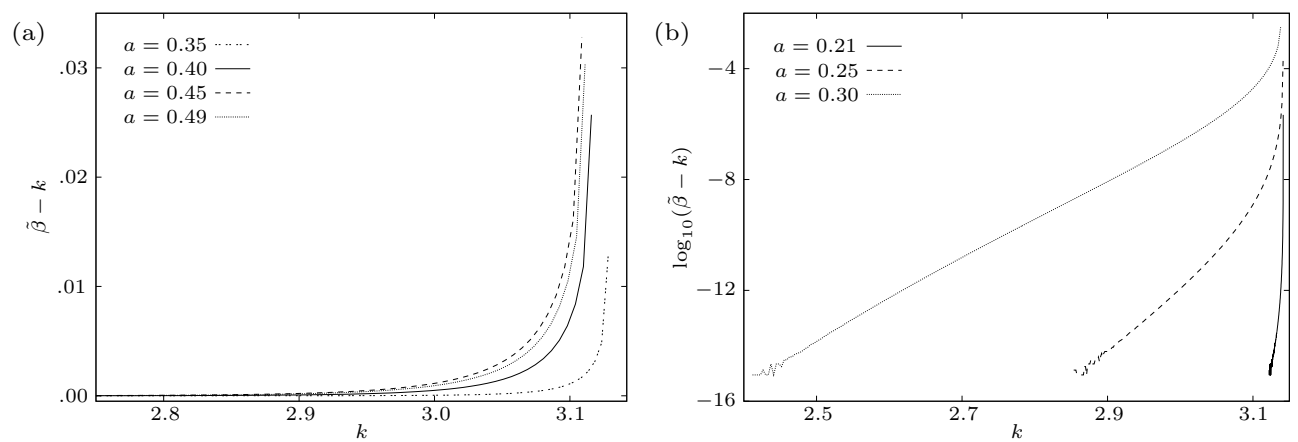

Fig. 2. (a) Difference between the Bloch wavenumber $\tilde{\beta}$ and the free-space wavenumber $k$ for selected values of the radius a. (b) Plot of $\log _{10}(\tilde{\beta}-k)$ versus $k$ for smaller spheres. In both figures, the problem is scaled so that the array has unit spacing $(s=1)$, the azimuthal mode number is zero, and Neumann boundary conditions are applied on the spheres' surfaces.

(a)
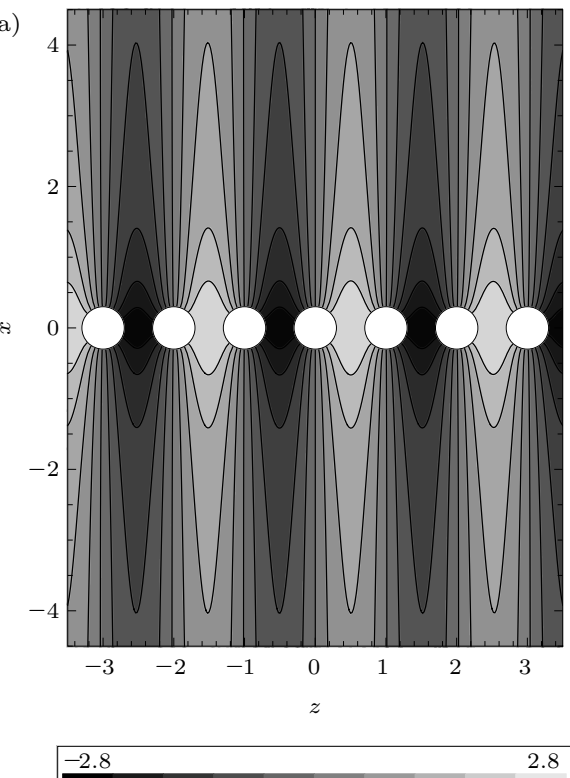

(b)
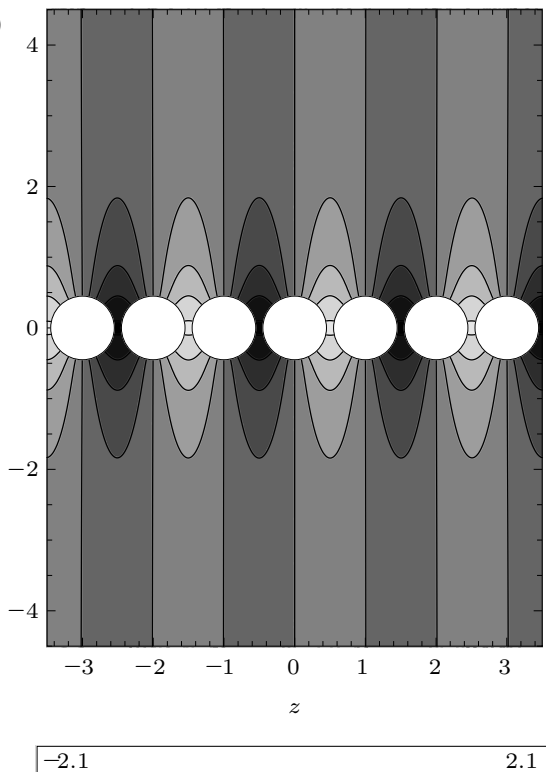

FIG. 3. Contour plot showing a cross section of a guided wave (azimuthal mode number zero, $k=3.1$ ) propagating along an array of rigid spheres with unit spacing and radius (a) $0.30,(\mathrm{~b}) 0.45$. The computed values of $\tilde{\beta}$ are approximately 3.100019 in (a) and 3.114056 in (b).

but in the latter case the trend is reversed for large $(a \gtrsim 0.46)$ spheres. Numerical evidence suggests that this wave exists for all sphere radii and all positive $k$ up to some $a$-dependent cut-off value $k_{\max }<\pi$, which occurs when $\tilde{\beta}=\pi$. However, $\tilde{\beta}-k$ decreases rapidly with both $k$ and $a$, to the point where the two wavenumbers cannot be distinguished by a machine working in double precision, as shown in Figure 2(b).

Figure 3 shows contour plots of $\operatorname{Re}\left[u^{0}(\mathbf{r})\right]$ in the plane $y=0$ for two guided waves with the same value for $k$ but different values for $a$. In both cases, the coefficients $A_{n}^{0}$ are scaled so that $\sum_{n=0}^{\infty}\left|A_{n}^{0}\right|^{2}=1$. Note that the exponential decay of the field as $|x|$ is increased is more rapid in Figure 3(b) than in 3(a). This corresponds to the fact that the value of $\tilde{\beta}-k$ is greater in Figure 3(b) due to the size of the spheres. 


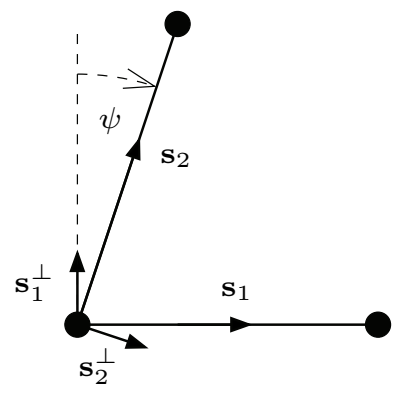

FIG. 4. The angle of skew $\psi$.

6. Two-dimensional array. In the two-dimensional case we write the subscripts on lattice vectors explicitly, and we must have $2 a \leq \min _{(j, p) \neq(0,0)} R_{j p}$ for all $j, p \in \mathbb{Z}$ so that the spheres do not overlap. A two-dimensional lattice may be described using any one of an infinite number of possible bases, but it has only a finite number of primitive (shortest possible) bases. For the basis $\left\{\mathbf{s}_{1}, \mathbf{s}_{2}\right\}$, we define the angle of skew $\psi$ via

$$
\mathbf{s}_{1} \cdot \mathbf{s}_{2}=s_{1} s_{2} \sin \psi
$$

We also define unit vectors $\mathbf{s}_{1}^{\perp}$ and $\mathbf{s}_{2}^{\perp}$ perpendicular to $\mathbf{s}_{1}$ and $\mathbf{s}_{2}$, respectively, so that $\mathbf{s}_{i}^{\perp} \cdot \mathbf{s}_{j} \geq 0$; see Figure 4 . Clearly, every lattice has bases for which

$$
s_{2} \geq s_{1} \quad \text { and } \quad s_{2} \sin |\psi|=\left|\hat{\mathbf{s}}_{1} \cdot \mathbf{s}_{2}\right| \leq s_{1} / 2 .
$$

In fact, by writing the vector $\mathbf{R}_{j p}$ in terms of $\hat{\mathbf{s}}_{1}$ and $\mathbf{s}_{1}^{\perp}$ one can easily show that these are the primitive bases. Note that the maximum possible skew for any primitive basis is $\pi / 6$, and this can occur only when $s_{1}=s_{2}$. Also, the vectors

$$
\mathbf{s}_{1}^{*}=\frac{\mathbf{s}_{2}^{\perp}}{s_{1} \cos \psi} \quad \text { and } \quad \mathbf{s}_{2}^{*}=\frac{\mathbf{s}_{1}^{\perp}}{s_{2} \cos \psi}
$$

form a primitive basis for the reciprocal lattice (see section 3), because $\mathbf{s}_{1}^{\perp} \cdot \mathbf{s}_{2}^{\perp}=$ $\sin (-\psi)$.

The principal region for $\boldsymbol{\beta}$, i.e., the two-dimensional analogue of the interval $(-\pi / s, \pi / s]$ in section 4 , is known as the first Brillouin zone, and we will denote this by $\mathcal{B}$. It consists of the set of points whose position vectors $\mathbf{q}$ satisfy the inequality

$$
q \leq\left|\mathbf{q}+\mathbf{R}_{m n}^{*}\right| \quad \text { for all } m, n \in \mathbb{Z} .
$$

In view of (3.3), guided waves of the form (3.6) are unchanged with the addition of an arbitrary reciprocal lattice vector to $\boldsymbol{\beta}$, and so no generality is lost in restricting our attention to $\boldsymbol{\beta} \in \mathcal{B}$. The method for constructing Brillouin zones is explained in [6, pp. 102-107] (see also [13, Appendix B]). Essentially, it consists of dividing the plane in two using the perpendicular bisector of the line from the origin to any other reciprocal lattice point, and then observing that the points in the half plane that does not contain the origin violate (6.4) and must therefore lie outside $\mathcal{B}$. By repeating this process for different reciprocal lattice points, one can eventually construct a polygon whose area is equal to that of a single reciprocal lattice cell $\left(4 \pi^{2}\left|\mathbf{s}_{1}^{*} \times \mathbf{s}_{2}^{*}\right|\right)$. It turns out that, at most, six perpendicular bisectors are required in the construction 


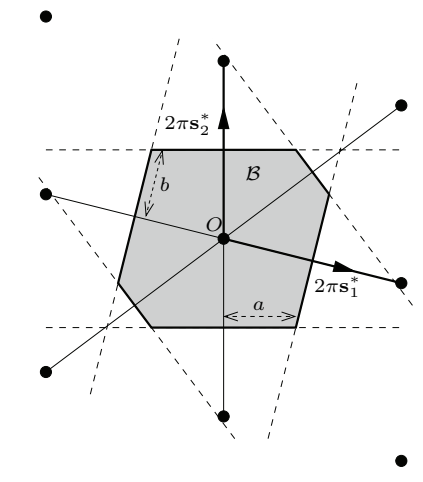

FIG. 5. The first Brillouin zone, $\mathcal{B}$. The dashed lines are the perpendicular bisectors used in the construction of the zone.

of $\mathcal{B}$, in which case it is a hexagonal region, as in Figure 5. Position vectors for the corner points of $\mathcal{B}$ can be determined by locating the intersections of the appropriate perpendicular bisectors. Assuming that $\left\{\mathbf{s}_{1}, \mathbf{s}_{2}\right\}$ is a primitive basis, we find that

$$
\mathbf{r}= \pm\left[\pi \mathbf{s}_{2}^{*} \pm g \hat{\mathbf{s}}_{1}\right] \quad \text { and } \quad \mathbf{r}= \pm\left[\pi \mathbf{s}_{1}^{*}+h \operatorname{sgn}(\psi) \hat{\mathbf{s}}_{2}\right],
$$

where

$$
g=\frac{\pi}{\cos \psi}\left(s_{1}^{*}-s_{2}^{*} \sin |\psi|\right) \quad \text { and } \quad h=\frac{\pi}{\cos \psi}\left(s_{2}^{*}-s_{1}^{*} \sin |\psi|\right) .
$$

Note that if $\psi=0$, then $\hat{\mathbf{s}}_{1}=\hat{\mathbf{s}}_{1}^{*}$ and $\hat{\mathbf{s}}_{2}=\hat{\mathbf{s}}_{2}^{*}$. In this case (6.5) defines only four distinct points and $\mathcal{B}$ reduces to a rectangular region.

Returning to the system of equations (3.10), we write the separation matrix explicitly using (A.14) to obtain

$$
A_{n}^{m}+4 \pi(-1)^{m} Z_{n} \sum_{\nu, \mu} A_{\nu}^{\mu} \sum_{\substack{q=|n-\nu| \\ n+\nu-q \text { even }}}^{n+\nu} \mathrm{i}^{n-\nu-q} \mathcal{G}(\nu, \mu ; n,-m ; q) \sigma_{q}^{\mu-m}(\boldsymbol{\beta})=0, \quad n \geq|m| .
$$

Here we have defined the two-dimensional lattice sum

$$
\sigma_{n}^{m}(\boldsymbol{\beta})=\sum_{\mathbf{R}_{j p} \in \Lambda}^{\prime} \mathrm{e}^{\mathrm{i} \boldsymbol{\beta} \cdot \mathbf{R}_{j p}} \mathrm{~h}_{n}\left(k R_{j p}\right) \mathrm{Y}_{n}^{m}\left(\hat{\mathbf{R}}_{j p}\right),
$$

where $\mathrm{Y}_{n}^{m}(\cdot)$ is a spherical harmonic (see the appendix). The properties of $\sigma_{n}^{m}$ depend upon the choice of coordinate system (see [21]), and these in turn affect the difficulty of our subsequent calculations. The reduction of (6.7) to a real system is simplest if the axis of the spherical polar coordinate system is chosen parallel to a basis vector, but in all other respects the problem turns out to be significantly simpler if the axis is perpendicular to the array. (Of course we can translate between the two systems using rotation matrices [23, Appendix C], but this is a complication that we prefer to avoid.) Therefore, we take the basis vectors to be

$$
\mathbf{s}_{1}=s_{1} \mathbf{e}_{x} \quad \text { and } \quad \mathbf{s}_{2}=\eta_{1} \mathbf{e}_{x}+\eta_{2} \mathbf{e}_{y}
$$

(see Figure 6) and employ spherical polar coordinates with $\mathbf{e}_{z}$ as the polar axis, so that $z=r \cos \theta$. In this case we have

$$
\sigma_{n}^{m} \equiv 0 \quad \text { if } n+m \text { is odd, }
$$

Copyright (c) by SIAM. Unauthorized reproduction of this article is prohibited. 


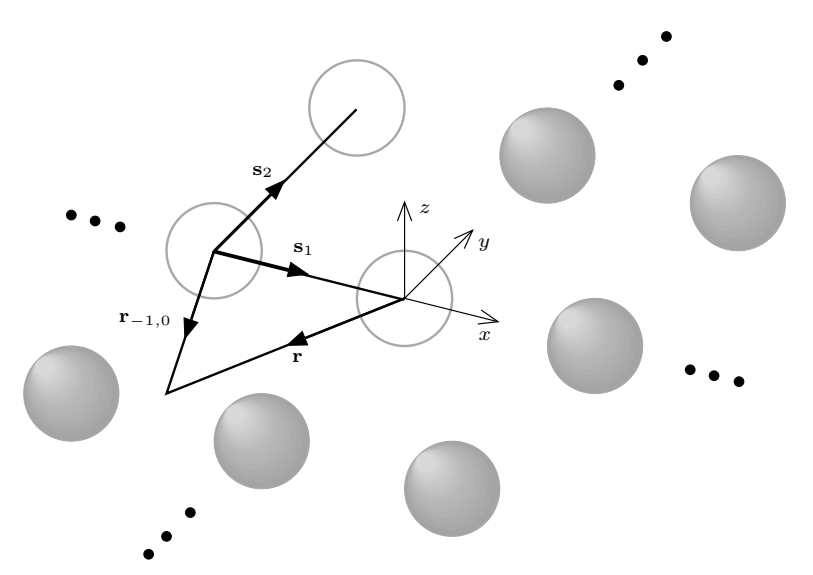

FIG. 6. Schematic diagram of a section of the two-dimensional array showing the coordinate system in use. The sphere centers are at $\mathbf{r}=\mathbf{R}_{j p}=j \mathbf{s}_{1}+p \mathbf{s}_{2}$, and a position vector relative to the center of sphere $(j, p)$ is defined via $\mathbf{r}_{j p}=\mathbf{r}-\mathbf{R}_{j p}$.

which follows from (A.3) and the fact that $\mathbf{R}_{j p}$ lies in the plane of the lattice.

A modal expansion analogous to (4.5) can now be derived by substituting the integral representation (A.10) into (3.6) and applying the Poisson summation formula (3.4). This final step is only possible with the coordinates chosen so that the polar axis is perpendicular to the array. We find that

$$
u(\mathbf{r})=\sum_{\mathbf{R}_{j p}^{*} \in \Lambda^{*}} \mathcal{M}_{j p} \mathrm{e}^{\mathrm{i} \cdot \boldsymbol{\beta}_{j p}} \mathrm{e}^{-k \gamma_{j p}|z|},
$$

where the mode amplitudes are given by

$$
\mathcal{M}_{j p}=-\frac{2 \pi \mathrm{i}}{k^{2} \mathcal{A} \gamma_{j p}} \sum_{n, m} \mathrm{i}^{n} A_{n}^{m} \lambda_{n m}(-\operatorname{sgn} z)^{n-m} \mathrm{e}^{\mathrm{i} m \alpha_{j p}} \mathrm{P}_{n}^{m}\left(\mathrm{i} \gamma_{j p}\right) .
$$

Here, $\lambda_{n m}$ and $\mathrm{P}_{n}^{m}(\cdot)$ are given by (A.6) and (A.1), respectively, and we have defined

$$
\boldsymbol{\beta}_{j p}=\boldsymbol{\beta}+\mathbf{R}_{j p}^{*}=\beta_{j p}\left(\cos \alpha_{j p} \mathbf{e}_{x}+\sin \alpha_{j p} \mathbf{e}_{y}\right) \quad \text { and } \quad \gamma_{j p}=\gamma\left(\beta_{j p} / k\right) ;
$$

the function $\gamma$ is defined in (A.11). If $\beta_{j p}>k$, then $\gamma_{j p}>0$, and if this is true for all pairs of integers $j$ and $p$, which holds for $\boldsymbol{\beta} \in \mathcal{B}$ when $\beta>k$, then (6.11) shows that $u(\mathbf{r})$ decays exponentially as $|z| \rightarrow \infty$, meaning that the radiation condition (3.7) is satisfied. In this case, we use the identity [21]

$$
2 \sigma_{n}^{m}=\sigma_{n}^{m}-\overline{\sigma_{n}^{-m}}-\delta_{n 0} \delta_{m 0} / \sqrt{\pi}
$$

along with (A.19), to simplify the system (6.7) to

$$
\begin{array}{r}
A_{n}^{m}-2 \pi \mathrm{i}(-1)^{m} W_{n} \sum_{\nu, \mu} A_{\nu}^{\mu} \sum_{\substack{q=|n-\nu| \\
n+\nu-q \text { even }}}^{n+\nu} \mathrm{i}^{n-\nu-q} \mathcal{G}(\nu, \mu ; n,-m ; q)\left(\sigma_{q}^{\mu-m}-\overline{\sigma_{q}^{m-\mu}}\right)=0, \\
n \geq|m|,
\end{array}
$$

Copyright $@$ by SIAM. Unauthorized reproduction of this article is prohibited. 
where $W_{n}$ is the quantity defined in (2.8). If we now replace $m$ and $\mu$ by $-m$ and $-\mu$, respectively, take the complex conjugate, and apply the symmetry relation (A.18), we obtain exactly the same system of equations for $\overline{A_{n}^{-m}}$, since $W_{n}$ is real. We can therefore discard the equations with $m<0$ and set $\overline{A_{n}^{-m}}=A_{n}^{m}$. Writing

$$
A_{n}^{m}=U_{n}^{m}+\mathrm{i} V_{n}^{m}
$$

and defining

$$
X_{n \nu}^{m \mu}+\mathrm{i} Y_{n \nu}^{m \mu}=2 \pi(-1)^{m} \sum_{\substack{q=|n-\nu| \\ n+\nu-q \text { even }}}^{n+\nu} \mathrm{i}^{n-\nu-q} \mathcal{G}(\nu, \mu ; n,-m ; q)\left(\sigma_{q}^{\mu-m}-\overline{\sigma_{q}^{m-\mu}}\right)
$$

with $U_{n}^{m}, V_{n}^{m}, X_{\nu n}^{\mu m}$, and $Y_{\nu n}^{\mu m}$ real, we obtain the real system of equations

$$
\begin{array}{ll}
U_{n}^{m}+\frac{W_{n}}{2} \sum_{\nu=0}^{\infty} \sum_{\mu=0}^{\nu} \epsilon_{\mu}\left[U_{\nu}^{\mu}\left(Y_{n \nu}^{m \mu}+Y_{n \nu}^{m,-\mu}\right)+V_{\nu}^{\mu}\left(X_{n \nu}^{m \mu}-X_{n \nu}^{m,-\mu}\right)\right]=0, & n \geq m \geq 0, \\
V_{n}^{m}-\frac{W_{n}}{2} \sum_{\nu=0}^{\infty} \sum_{\mu=0}^{\nu} \epsilon_{\mu}\left[U_{\nu}^{\mu}\left(X_{n \nu}^{m \mu}+X_{n \nu}^{m,-\mu}\right)-V_{\nu}^{\mu}\left(Y_{n \nu}^{m \mu}-Y_{n \nu}^{m,-\mu}\right)\right]=0, & n \geq m \geq 0,
\end{array}
$$

where $\epsilon_{n}=2-\delta_{n 0}$. Now (6.10) and (6.17) show that $X_{n \nu}^{m \mu} \equiv Y_{n \nu}^{m \mu} \equiv 0$ if $n+m+\nu+\mu$ is odd, meaning that (6.18) can be decoupled into two subsystems, one in which $n+m$ and $\nu+\mu$ are even and another in which $n+m$ and $\nu+\mu$ are odd. Equation (A.9) shows that solutions to these are symmetric and antisymmetric about the plane of the array, respectively. Note that the symmetric problem is equivalent to the case of a rigid plane embossed with a periodic array of hemispherical bumps.

As in the one-dimensional case, the lattice sums are singular when $\beta=k$. Thus, from [21], we have

$$
\sigma_{n}^{m}=\widehat{\sigma}_{n}^{m}+\frac{2 \pi \mathrm{i}^{n-1} \mathrm{Y}_{n}^{m}(\hat{\boldsymbol{\beta}})}{k \mathcal{A} \sqrt{\beta^{2}-k^{2}}}
$$

where $\widehat{\sigma}_{n}^{m}$ remains bounded as $\beta \rightarrow k$. Making use of (A.7), (6.17) becomes

$$
X_{n \nu}^{m \mu}+\mathrm{i} Y_{n \nu}^{m \mu}=a_{n \nu}^{m \mu}+2 \pi(-1)^{m} \sum_{\substack{q=|n-\nu| \\ n+\nu-q \text { even }}}^{n+\nu} \mathrm{i}^{n-\nu-q} \mathcal{G}(\nu, \mu ; n,-m ; q)\left(\widehat{\sigma}_{q}^{\mu-m}-\overline{\widehat{\sigma}_{q}^{m-\mu}}\right),
$$

where

$$
a_{n \nu}^{m \mu}=\frac{8 \pi^{2} \mathrm{i}^{n-\nu+2 m-1}}{k \mathcal{A} \sqrt{\beta^{2}-k^{2}}} \mathrm{Y}_{n}^{-m}(\hat{\boldsymbol{\beta}}) \mathrm{Y}_{\nu}^{\mu}(\hat{\boldsymbol{\beta}}) .
$$

Here we have again used the linearization formula for spherical harmonics [23, Theorem 3.20] to simplify the expression for the coefficient of the singular term. Note that $a_{n \nu}^{m \mu}$ vanishes identically from the antisymmetric subsystem (in which $n+m$ is odd) because $\boldsymbol{\beta}$ lies in the plane $z=0$ (see (A.3)). 
7. Numerical results for two-dimensional arrays. There are a number of parameters that determine the geometry, and some care must be taken so as to ensure that all possibilities are included but none repeated. Clearly, we can assume without loss of generality that $\mathbf{s}_{1}=[1,0]$ and $s_{2} \geq 1$ and $\eta_{2}=\mathbf{e}_{y} \cdot \mathbf{s}_{2}>0$. Moreover, since the existence of a mode at $\boldsymbol{\beta}=\boldsymbol{\beta}_{0}$ implies the existence of a mode with $\boldsymbol{\beta}=-\boldsymbol{\beta}_{0}$, we may restrict our attention to cases where $\eta_{1}=\mathbf{e}_{x} \cdot \mathbf{s}_{2} \geq 0$ (i.e., the lattice has nonnegative skew), provided we consider all possible values for $\boldsymbol{\beta} \in \mathcal{B}$. Under these assumptions, each lattice has a unique primitive basis that satisfies (6.2). Compared with the linear array example, the idea of a cut-off (i.e., a maximum value for $k$ ) is now more subtle, because modes may propagate in some directions but not others. In particular, if we write $\boldsymbol{\beta}=\beta[\cos \alpha, \sin \alpha]$, then we clearly must have

$$
k<\beta \leq \mathrm{b}(\alpha),
$$

where $\mathrm{b}(\alpha)$ is the distance to the edge of the Brillouin zone in the $\alpha$-direction. The absolute cut-off, at which the circular region $\beta \leq k$ completely covers the Brillouin zone, occurs at

$$
k=\frac{\pi}{\cos ^{2} \psi} \sqrt{\frac{1}{s_{1}^{2}}+\frac{1}{s_{2}^{2}}-\frac{2 \sin |\psi|}{s_{1} s_{2}}},
$$

which is the distance from the origin to the corners of $\mathcal{B}$ (see (6.5)).

The basic configuration of the numerical code used to search for modes is the same as in the linear array case except that the method of reduction in lattice dimension described in [21] is now used to compute the lattice sums. Comprehensive searches for this case are rather demanding due to the number of variables and the complexity of the lattice sums. Nevertheless, by using multiple machines simultaneously, we were able to systematically run parameter sweeps across the full ranges of the bounded parameters $\psi, a, k$, and $\alpha$ for $1 \leq s_{2} \leq 5$. No modes were found in the Dirichlet case, again consistent with known results in two dimensions.

A two-dimensional array of spheres is a more substantial structure than a linear array and, perhaps unsurprisingly, it is much easier to find guided modes in this case (in the sense that the difference between $\beta$ and $k$ is typically much greater than in the examples considered in section 5). In particular, there is no difficulty in finding a Neumann mode symmetric about the plane of the array for small $a$ and/or small $k$. The situation appears to be much more akin to the two-dimensional problem of surface waves propagating along an array of rigid circular cylinders, as can be seen by comparing the results presented in Figure 7(a), which shows dispersion curves relating $k$ and $\beta$ for a square lattice and various values of $a$, to those of Figure 10 of [9]. Nevertheless, the trends observed in the linear array case (i.e., increasing $\beta-k$ with both $a$ and $k$ ) are again visible here. However, the simple behavior evident in Figure 7(a) (i.e., a single solution for each $\alpha$ and $k$ below the cut-off) is not ubiquitous. A number of "exotic" effects can occur when $k \approx \mathrm{b}(\alpha)$, especially in situations with low symmetry. An example is given in Figure $7(\mathrm{~b})$. Here there is a single mode for small $k$, but this disappears before $\beta$ reaches $\mathrm{b}(\alpha)$. At a slightly higher frequency a second mode cuts on and a third enters via the Brillouin zone edge. These finally come together and cut off at $k \approx 3.298$.

We have also found modes that are antisymmetric about the plane of the array, but only when $a \gtrsim 0.467$. The range of lattices for which these occur increases with $a$; when $a=0.467$, modes were found only when $s_{2}=s_{1}$ and $\psi=\pi / 6$ (the densest 
(a)

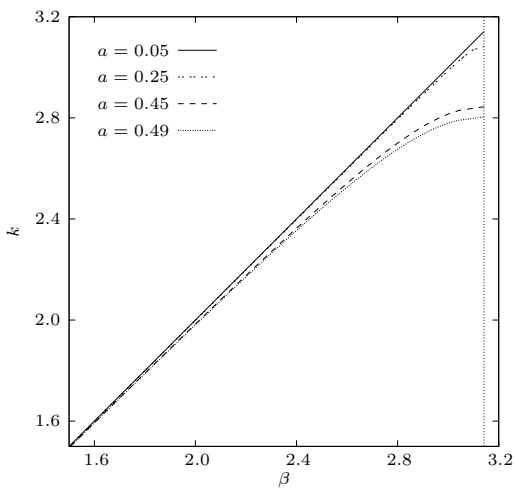

(b)

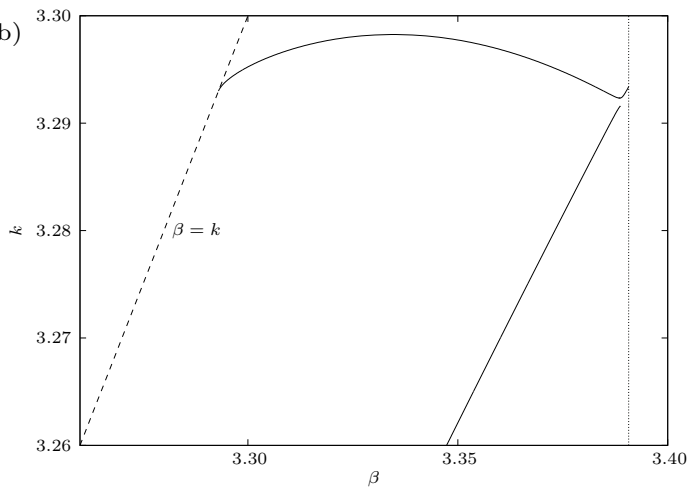

FIG. 7. (a) Plots showing the Bloch wavenumber $\beta$ and the free-space wavenumber $k$ for $\mathbf{s}_{2}=$ $[0,1]$ and $\alpha=0$ at selected radii. (b) Plot showing the Bloch wavenumber $\beta$ and the free-space wavenumber $k$ for $s_{2}=1.8, \psi \approx 0.08960 \pi$, and $\alpha=0.18 \pi$ with $a=0.49$. In both cases, the dotted vertical line marks the edge of the Brillouin zone.

possible packing of the spheres having fixed $s_{1}=1$ and assumed that $s_{2} \geq s_{1}$ ). The fact that antisymmetric modes do not exist for smaller spheres is consistent with the the two-dimensional cylinder problem [9], though there is a distinction between these problems that should be noted. In the two-dimensional case, as the radius increases, the symmetric and antisymmetric modes coalesce due to the fact that when adjacent cylinders touch, the two sides of the array become disconnected (see, for example, Figure 2.2 of [20]). This is clearly not the case for an array of spheres.

Figures $8(\mathrm{a})-(\mathrm{d})$ display a sequence of plots showing dispersion curves relating the frequency $k$ and the Bloch vector $\boldsymbol{\beta}$ for symmetric surface modes. The geometry is that of a skew lattice with $s_{2}=1.2, \psi=\pi / 10$, and $a=0.45$. These parameters were chosen because they lead to relatively large values of $\beta-k$, but the qualitative behavior is fairly typical. The dots at the center of the shaded circles are points in the reciprocal lattice. The difference between the figures is the value of $k$, which ranges from 2.45 in Figure 8(a) to 3.45 in Figure 8(d). The central shaded circle shows the region $\beta \leq k$, which is excluded by virtue of (7.1), and the dashed line shows the boundary of the Brillouin zone. The dispersion curves therefore lie between this circle and the dashed line, repeated throughout the plane via periodicity. Although the dispersion curves lie close to the boundary of the shaded circles, the general trend for $\beta-k$ to increase with $k$ is still visible. For this particular lattice the absolute cut-off occurs at $k \approx 3.772$. Isofrequency diagrams such as these can be used to determine the direction in which the energy associated with a particular mode propagates along the array. As described in [13, pp. 40 and 223] the energy propagates in the direction of the group velocity, which is normal to the dispersion curves (in the direction of increasing $k$ ). The quantity $\alpha(=\arg \boldsymbol{\beta})$, on the other hand, has no simple physical interpretation. Thus, in Figures 8(a), (b), and (d), due to the closed nature of the dispersion curves, modes which propagate in any direction are possible, but for $k=2.75$ as in Figure 8 (b) this is not the case. Here, modes with group velocity predominantly in the $y$-direction are forbidden. We have observed the pattern in Figure 8(b) only for stretched lattices (i.e., those with $s_{2}>1$ ). In fact, from (6.3) and Figure 5 it is clear that increasing $s_{2}$ squeezes the Brillouin zone down to a thin strip aligned with the $x$-axis, and so if we let $s_{2} \rightarrow \infty$, we can cut off all modes except those with group velocity in the $x$-direction. 

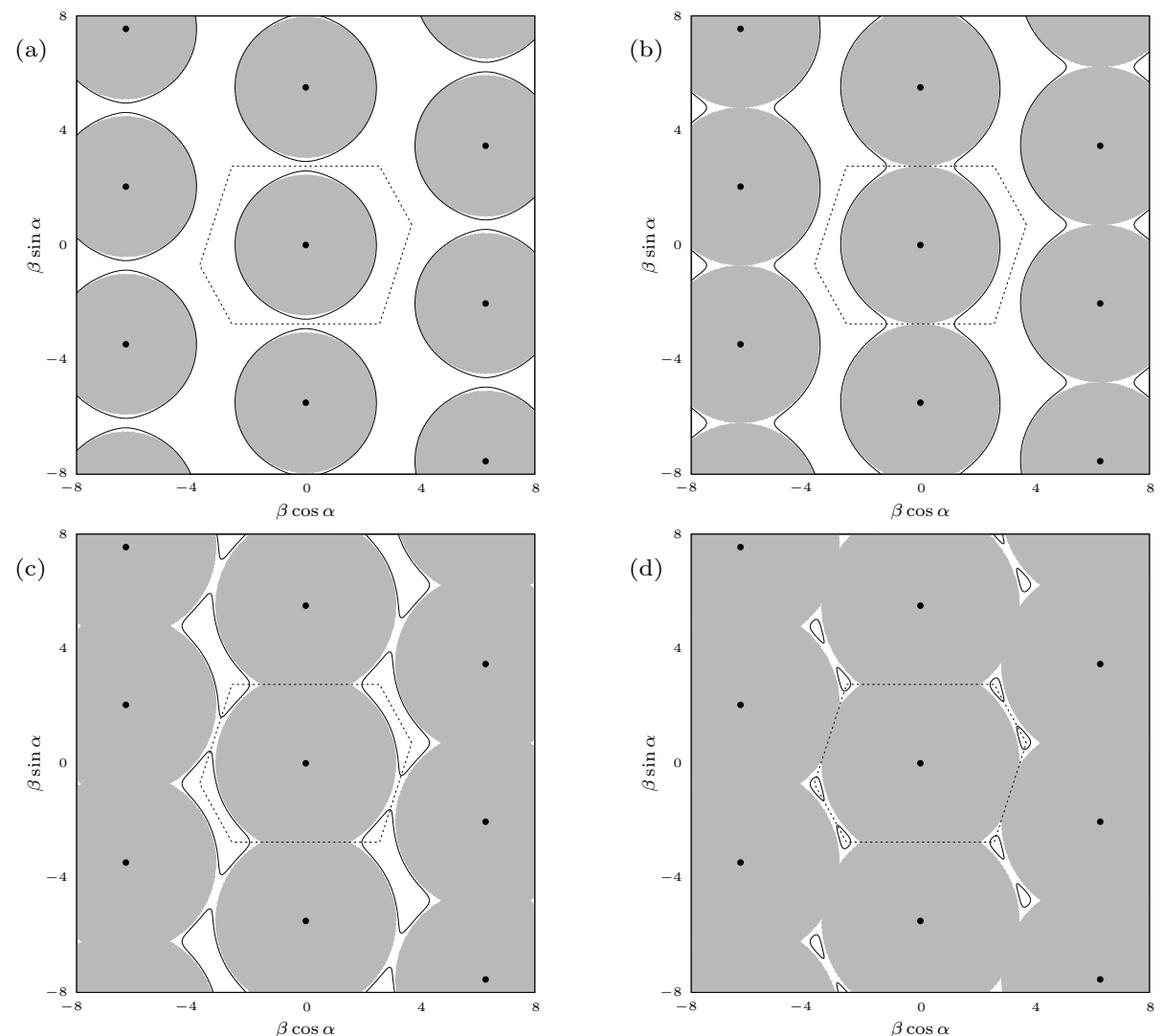

FIG. 8. Contour plots showing the location of the Bloch wavevector $\boldsymbol{\beta}$ for symmetric modes (solid lines) in the plane of the reciprocal lattice, with $s_{2}=1.2, \psi=\pi / 10, a=0.45$, and (a) $k=2.45$, (b) $k=2.75$, (c) $k=3.15$, (d) $k=3.45$. The dotted line shows the boundary of the Brillouin zone, and the shaded circles (which have radius $k$ ) cover the regions in which $\beta_{j p} \leq k$ for some $j, p \in \mathbb{Z}$.

A situation similar to that shown in Figure 8(b) is shown in Figure 9. The sphere radius is smaller than in the previous figure as this leads to more informative contour plots. Figure $9(\mathrm{a})$ is an isofrequency diagram showing the dispersion curves for an array with $s_{2}=1.2, \psi=\pi / 10, a=0.25$, and $k=3.0$. The magnitude of $\beta-k$ is very small along the whole dispersion curve in this case. Figures $9(\mathrm{~b})-(\mathrm{d})$ show contour plots of $\operatorname{Re}[u(\mathbf{r})]$ in the plane $z=0$ (the coefficients are normalized so that $\sum_{n, m}\left|A_{n}^{m}\right|^{2}=1$ ) for the modes corresponding to the three points marked in the first figure. The normals to the dispersion curve at (b) and at (c) are parallel, and so the corresponding modes propagate in the same direction (parallel to the $x$-axis). This is clearly seen from the accompanying mode plots. On the other hand Figures 9(c) and (d) illustrate two modes propagating at markedly different angles, despite the minimal change in $\alpha$. This is related to the superprism effect discussed in [13, p. 225].

Figure 10 shows isofrequency contours for antisymmetric modes for an array with $s_{2}=1, \psi=\pi / 6, a=0.50$, so that the spheres are as closely packed as possible with each sphere in contact with six others. The fact that the spheres are touching each other does not have a significant qualitative effect (one might guess that this is the case because the spheres touch in the plane $z=0$, where $u=0$ for an antisymmetric 

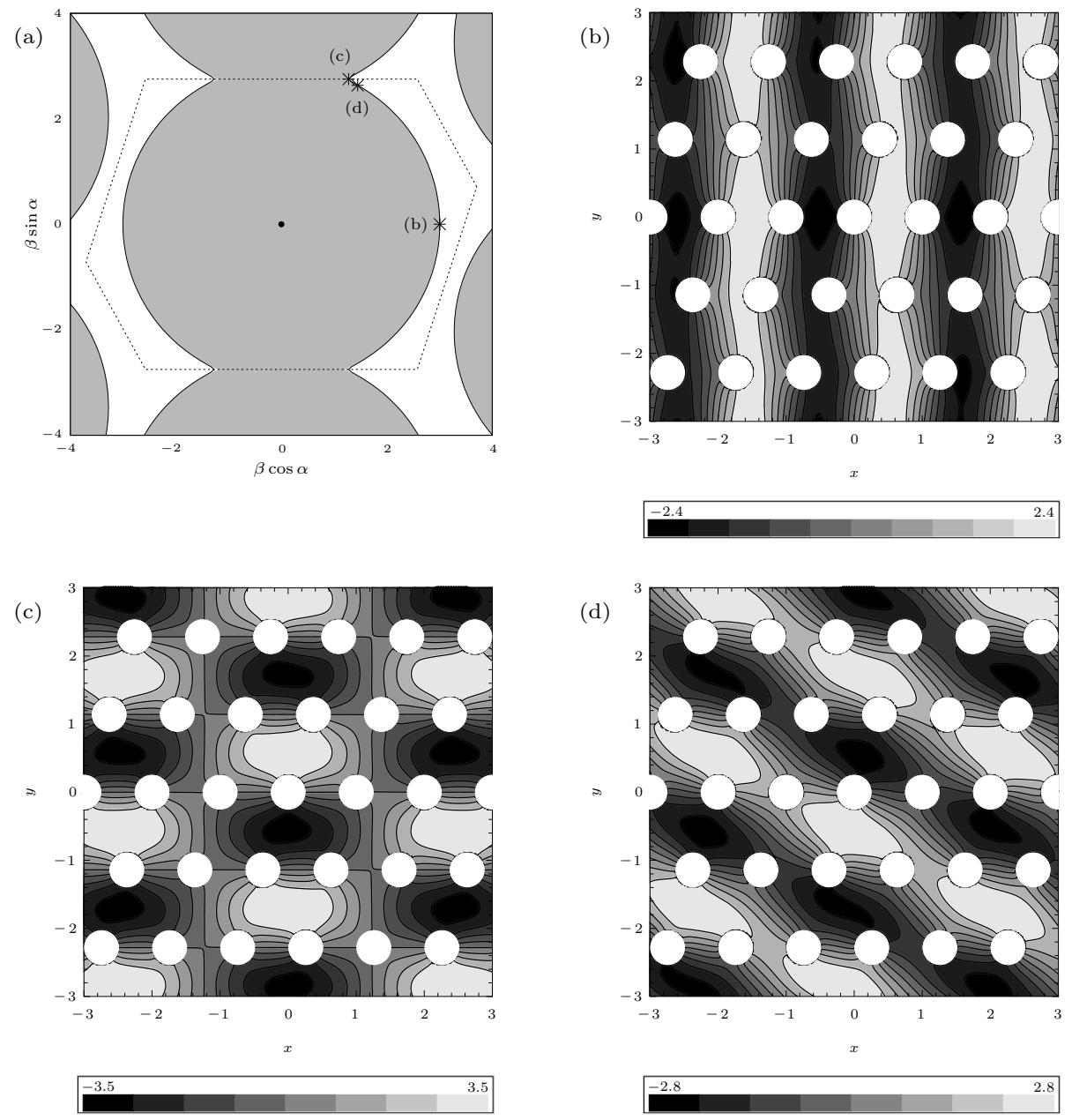

FIG. 9. Contour plots with $s_{2}=1.2, \psi=\pi / 10, a=0.25$, and $k=3.0$. (a) shows the location of the Bloch wavevector $\boldsymbol{\beta}$ for symmetric modes (solid lines) in the plane of the reciprocal lattice. (b)-(d) show $\operatorname{Re}[u(\mathbf{r})]$ with $z=0$ for the values of $\boldsymbol{\beta}$ indicated by crosshairs in (a); thus (b) $\boldsymbol{\beta} \approx[3.004,0],(\mathrm{c}) \boldsymbol{\beta} \approx[1.275,2.753]$, (d) $\boldsymbol{\beta} \approx[1.449,2.635]$.

mode). The frequencies illustrated range from $k=3.5$ to $k=3.8$, and the dispersion curves shown are typical for antisymmetric modes. The directions in which surface waves can propagate in this case change significantly as $k$ is varied, and in Figure 10(d) the permitted propagation directions are predominantly in a direction that is parallel to a lattice vector.

8. Conclusion. In this article we have studied guided acoustic waves propagating along one- and two-dimensional arrays of rigid spheres. In each case these modes correspond to quasi-periodic solutions to the appropriate boundary-value problem. The quasi-periodicity introduces a cut-off frequency, below which waves cannot radiate away from the array, and we search below this cut-off for the existence of guided modes.

In the case of a one-dimensional array we find that modes which are axisymmetric do exist, though the difference between the frequency parameter $k$ and the Bloch 

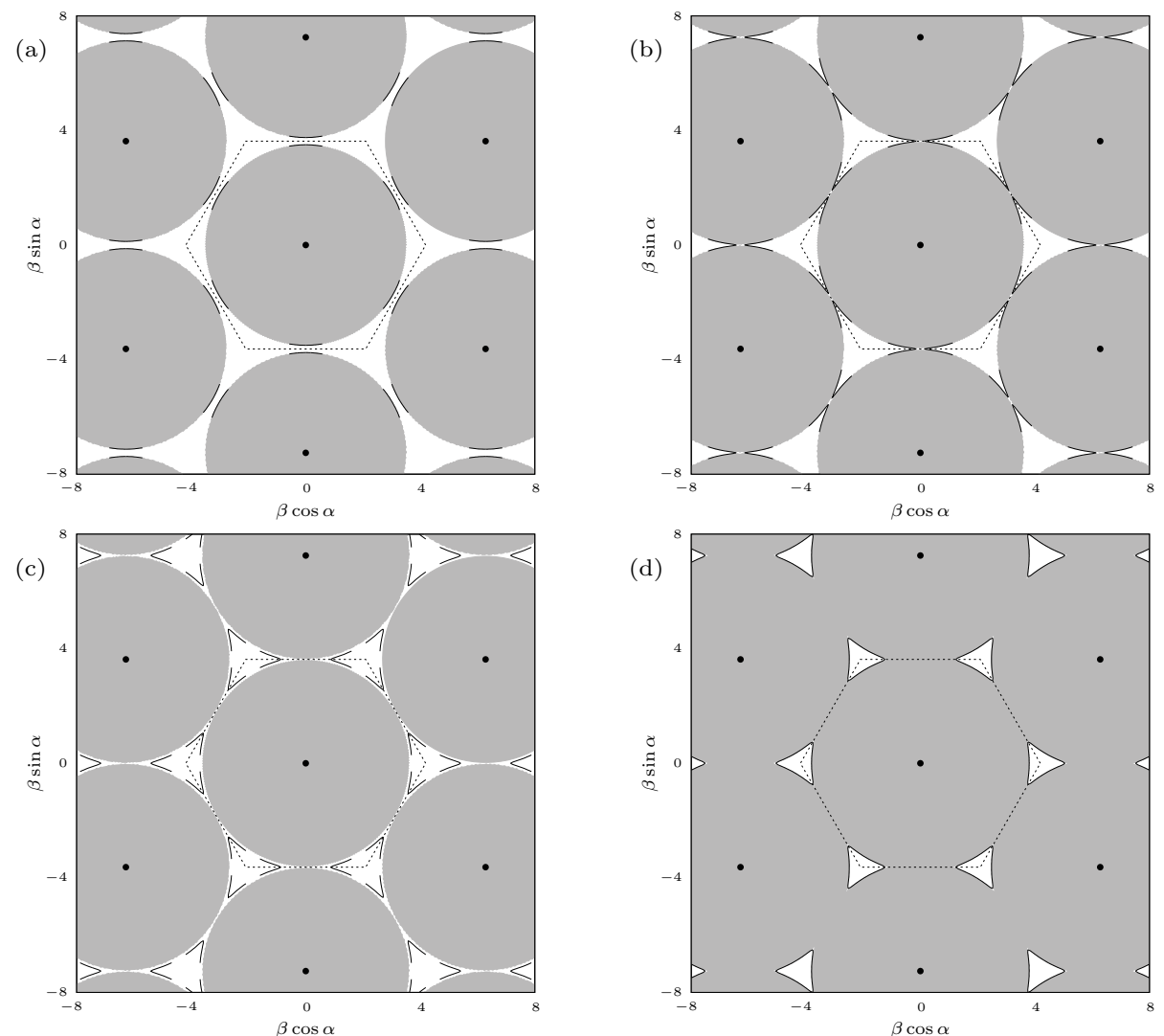

FIG. 10. Contour plots showing the location of $\boldsymbol{\beta}$ for antisymmetric modes (solid lines) in the plane of the reciprocal lattice, with $s_{2}=1, \psi=\pi / 6, a=0.50$, and (a) $k=3.5$, (b) $k=3.6$, (c) $k=3.7$, (d) $k=3.8$. The dotted line shows the boundary of the Brillouin zone, and the shaded circles (which have radius $k$ ) cover the regions in which $\beta_{j p} \leq k$ for some $j, p \in \mathbb{Z}$.

parameter $\tilde{\beta}$ is very small and can be difficult to determine numerically, particularly when the spheres are small. Previous work on the excitation of surface modes in a related two-dimensional problem [38] suggests that, as a consequence, these modes will be difficult to excite.

For two-dimensional arrays, we have computed modes that are either symmetric or antisymmetric about the plane of the array (the symmetric case is equivalent to hemispherical protrusions on a rigid plane), and we have considered arbitrary skew lattices. The dispersion curves have an extremely rich structure as a function of the various parameters which govern the problem. One of our key findings is that the possible directions in which modes can propagate vary with frequency. This suggests the possibility of constructing periodically embossed surfaces which channel energy to infinity in some directions but not in others.

We have not attempted to provide a mathematical proof of the existence of the modes which we have computed, nor to prove that in the case of sound-soft spheres modes do not exist. These proofs do exist for related two-dimensional problems (see, for example, $[15,5,36,18]$ ), and we are currently working on extending these to cover the problems treated in this article. 
Appendix. Spherical harmonics and wave functions. The associated Legendre function is defined here, for nonnegative order and $|x| \leq 1$, by

$$
\mathrm{P}_{n}^{m}(x)=\left(1-x^{2}\right)^{m / 2} \frac{\mathrm{d}^{m}}{\mathrm{~d} x^{m}} \mathrm{P}_{n}(x)=\frac{\left(1-x^{2}\right)^{m / 2}}{2^{n} n !} \frac{\mathrm{d}^{m+n}}{\mathrm{~d} x^{m+n}}\left(x^{2}-1\right)^{n}, \quad n \geq m \geq 0 .
$$

This is the convention adopted in [23]. Some authors include an extra factor of $(-1)^{m}$ (often referred to as the Condon-Shortley phase). If $m>n$, then $\mathrm{P}_{n}^{m}(x) \equiv 0$. The extension to negative order is accomplished via

$$
\mathrm{P}_{n}^{-m}(x)=(-1)^{m} \frac{(n-m) !}{(n+m) !} \mathrm{P}_{n}^{m}(x), \quad n \geq|m|,
$$

and we note that

$$
\mathrm{P}_{n}^{m}(0)=0 \quad \text { for } n+m \text { odd }
$$

We refer to a solution of the Helmholtz equation as a wave function. In particular we use the notation

$$
\mathcal{J}_{n}^{m}(\mathbf{r})=\mathrm{j}_{n}(k r) \mathrm{Y}_{n}^{m}(\hat{\mathbf{r}}) \quad \text { and } \quad \mathcal{H}_{n}^{m}(\mathbf{r})=\mathrm{h}_{n}(k r) \mathrm{Y}_{n}^{m}(\hat{\mathbf{r}})
$$

for, respectively, regular and outgoing spherical wave functions. Here $\mathrm{j}_{n}(\cdot)$ and $\mathrm{h}_{n}(\cdot) \equiv \mathrm{h}_{n}^{(1)}(\cdot)$ are, respectively, spherical Bessel functions of the first kind and spherical Hankel functions of the first kind, and $\mathrm{Y}_{n}^{m}$ are spherical harmonics defined by

$$
\mathrm{Y}_{n}^{m}(\hat{\mathbf{r}}) \equiv \mathrm{Y}_{n}^{m}(\theta, \phi)=(-1)^{m} \lambda_{n m} \mathrm{P}_{n}^{m}(\cos \theta) \mathrm{e}^{\mathrm{i} m \phi}, \quad n \geq|m| \geq 0,
$$

where

$$
\lambda_{n m}=\sqrt{\frac{(2 n+1)(n-m) !}{4 \pi(n+m) !}} .
$$

We note that

$$
\overline{\mathrm{Y}_{n}^{m}(\hat{\mathbf{r}})}=(-1)^{m} \mathrm{Y}_{n}^{-m}(\hat{\mathbf{r}}), \quad \mathrm{Y}_{n}^{m}(-\hat{\mathbf{r}})=(-1)^{n} \mathrm{Y}_{n}^{m}(\hat{\mathbf{r}}),
$$

and

$$
\int_{\Omega} \mathrm{Y}_{n}^{m} \overline{\mathrm{Y}_{\nu}^{\mu}} \mathrm{d} \Omega=\delta_{n \nu} \delta_{m \mu}
$$

the integral being over the surface of the unit sphere. Also,

$$
\mathrm{Y}_{n}^{m}(\theta, \phi)=(-1)^{n+m} \mathrm{Y}_{n}^{m}(\pi-\theta, \phi) .
$$

Wave functions may be represented as Fourier integrals, and these representations are often useful in conjunction with the Poisson summation formula (3.4). Using spherical polar coordinates with $z=r \cos \theta$ as in sections 4 and 6 , we have [17]

$$
\mathrm{h}_{n}(k r) \mathrm{P}_{n}^{m}(\cos \theta) \mathrm{e}^{\mathrm{i} m \phi}=\frac{(\operatorname{sgn} z)^{n-m}}{2 \pi \mathrm{i}^{n+1}} \int_{-\infty}^{\infty} \int_{-\infty}^{\infty} \mathrm{e}^{\mathrm{i} m \alpha} \mathrm{e}^{\mathrm{i} k \mathbf{r} \cdot \mathbf{q}} \mathrm{e}^{-k \gamma(q)|z|} \mathrm{P}_{n}^{m}(\mathrm{i} \gamma(q)) \frac{\mathrm{d} q_{1} \mathrm{~d} q_{2}}{\gamma(q)},
$$

where $q_{1}=q \cos \alpha$ and $q_{2}=q \sin \alpha$, and we have introduced the function

$$
\gamma(z)=\left(z^{2}-1\right)^{1 / 2}, \quad \gamma(0)=-\mathrm{i},
$$

Copyright (c) by SIAM. Unauthorized reproduction of this article is prohibited. 
with infinite branch cuts placed along $z= \pm(1+\mathrm{i} u), u>0$. For real argument $\gamma$ is either positive real or negative imaginary. Note that this definition serves to analytically continue (A.1) (i.e., by writing $\left.\left(1-z^{2}\right)^{1 / 2}=\mathrm{i} \gamma(z)\right)$, but the resulting function which appears in (A.10) differs from the usual associated Legendre function found in books since the branch cut is normally taken along the interval $(-1,1)$. Alternatively, in cylindrical polar coordinates [17]

$$
\mathrm{h}_{n}(k r) \mathrm{P}_{n}^{m}(\cos \theta)=\frac{(-\mathrm{i})^{n+1}}{\pi} \int_{-\infty}^{\infty} \mathrm{e}^{\mathrm{i} k z t} \mathrm{~K}_{m}\left(k \sqrt{x^{2}+y^{2}} \gamma(t)\right) \mathrm{P}_{n}^{m}(t) \mathrm{d} t,
$$

in which $\mathrm{K}_{m}(\cdot)$ is a modified Bessel function.

It is possible to expand an outgoing wave function as a series of regular wave functions with a different origin. Thus, from [23, Theorem 3.27] we have, for $|\mathbf{a}|<|\mathbf{b}|$,

$$
\mathcal{H}_{n}^{m}(\mathbf{c})=\sum_{\nu, \mu} S_{n \nu}^{m \mu}(\mathbf{b}) \mathcal{J}_{\nu}^{\mu}(\mathbf{a})
$$

in which $\mathbf{c}=\mathbf{a}+\mathbf{b}$, and

$$
S_{n \nu}^{m \mu}(\mathbf{b})=4 \pi \mathrm{i}^{\nu-n}(-1)^{m} \sum_{\substack{q=|n-\nu| \\ n+\nu-q \text { even }}}^{n+\nu} \mathrm{i}^{q} \mathrm{~h}_{q}(k b) \overline{\mathrm{Y}_{q}^{\mu-m}(\hat{\mathbf{b}})} \mathcal{G}(n, m ; \nu,-\mu ; q) .
$$

Here $\mathcal{G}$ is a Gaunt coefficient defined by

$$
\mathcal{G}(n, m ; \nu, \mu ; q)=\int_{\Omega} \mathrm{Y}_{n}^{m} \mathrm{Y}_{\nu}^{\mu} \overline{\mathrm{Y}_{q}^{m+\mu}} \mathrm{d} \Omega,
$$

the integration being over the surface of the unit sphere. The matrix $\left[S_{n \nu}^{m \mu}\right]$ is known variously as the separation matrix, the translation matrix, or the propagator matrix. In view of (A.7), this satisfies the symmetry relationship

$$
S_{\nu n}^{\mu m}(-\mathbf{b})=(-1)^{n+\nu} S_{\nu n}^{\mu m}(\mathbf{b}) .
$$

For the special case in which $\mathbf{b}=\tilde{b} \mathbf{e}_{z}$ lies along the axis of the spherical coordinate system, we have [23, eqn. 3.88]

$$
S_{n \nu}^{m \mu}\left(\tilde{b} \mathbf{e}_{z}\right)=\sqrt{4 \pi} \delta_{m \mu} \mathrm{i}^{\nu-n}(-1)^{m} \sum_{\substack{q=|n-\nu| \\ n+\nu-q \text { even }}}^{n+\nu} \sqrt{2 q+1}(\mathrm{i} \operatorname{sgn} \tilde{b})^{q} \mathrm{~h}_{q}(k|\tilde{b}|) \mathcal{G}(n, m ; \nu,-m ; q) .
$$

A number of properties of the Gaunt coefficients follow directly from the definition (A.15) and (A.7) (see [23, p. 84]). In particular, $\mathcal{G}$ is real, and

$$
\mathcal{G}(n, m ; \nu, \mu ; q)=\mathcal{G}(n,-m ; \nu,-\mu ; q) .
$$

For certain combinations of parameters, $\mathcal{G}$ takes a simple form (see [23, Chapter 3] and [11]). In particular, when $q=0$ we can evaluate the integral using (A.8), to obtain

$$
\mathcal{G}(n, m ; \nu,-\mu ; 0)=(-1)^{m} \delta_{n \nu} \delta_{m \mu} / \sqrt{4 \pi}
$$




\section{REFERENCES}

[1] J. D. Achenbach And M. Kitahara, Reflection and transmission of an obliquely incident wave by an array of spherical cavities, J. Acoust. Soc. Amer., 80 (1986), pp. 1209-1214.

[2] J. D. Achenbach And M. Kitahara, Harmonic waves in a solid with a periodic distribution of spherical cavities, J. Acoust. Soc. Amer., 81 (1987), pp. 595-598.

[3] A. Alù And N. Engheta, Theory of linear chains of metamaterial/plasmonic particles as subdiffraction optical nanotransmission lines, Phys. Rev. B, 74 (2006), article 205436.

[4] A. Boag, Y. Leviatan, AND A. Boag, Analysis of electromagnetic scattering from linear periodic arrays of perfectly conducting bodies using a cylindrical current model, IEEE Trans. Antennas Propagat., 39 (1991), pp. 1332-1337.

[5] A.-S. Bonnet-Bendhia And F. Starling, Guided waves by electromagnetic gratings and nonuniqueness examples for the diffraction problem, Math. Methods Appl. Sci., 17 (1994), pp. 305-338.

[6] L. Brillouin, Wave Propagation in Periodic Structures, 2nd ed., Dover, New York, 1953.

[7] P. Etchegoin and R. T. Phillips, Photon focusing, internal diffraction, and surface states in periodic dielectric structures, Phys. Rev. B, 53 (1996), pp. 12674-12683.

[8] D. V. Evans and C. M. Linton, Edge waves along periodic coastlines, Quart. J. Mech. Appl. Math., 46 (1993), pp. 642-656.

[9] D. V. Evans And R. Porter, Trapping and near-trapping by arrays of cylinders in waves, J. Engrg. Math., 35 (1999), pp. 149-179.

[10] D. V. Evans AND R. Porter, On the existence of embedded surface waves along arrays of parallel plates, Quart. J. Mech. Appl. Math., 55 (2002), pp. 481-494.

[11] J. A. Gaunt, The triplets of helium, Philos. Trans. Roy. Soc. London Ser. A, 228 (1929), pp. 151-196.

[12] D. S. Janning And B. A. Munk, Effects of surface waves on the currents of truncated periodic arrays, IEEE Trans. Antennas Propagat., 50 (2002), pp. 1254-1265.

[13] J. D. Joannopoulos, S. G. Johnson, J. N. Winn, and R. D. Meade, Photonic crystals. Molding the Flow of Light, 2nd ed., Princeton University Press, Princeton, NJ, 2008.

[14] V. K. Kinra, B. K. Henderson, and K. I. Maslov, Elastodynamic response of layers of spherical particles in hexagonal and square periodic arrangements, J. Mech. Phys. Solids, 47 (1999), pp. 2147-2170.

[15] A. KIRSCH, Diffraction by periodic structures, in Inverse Problems in Mathematical Physics. Proceedings of the Lapland Conference on Inverse Problems (Saariselkä, Finland, 1992), L. Päivärinta and E. Somersalo, eds., Springer-Verlag, Berlin, 1993, pp. 87-102.

[16] A. F. Koenderink And A. Polman, Complex response and polariton-like dispersion splitting in periodic metal nanoparticle chains, Phys. Rev. B, 74 (2006), article 033402.

[17] C. M. Linton, Lattice sums for the Helmholtz equation, SIAM Rev., 52 (2010), pp. 630-674.

[18] C. M. Linton And M. McIver, The existence of Rayleigh-Bloch surface waves, J. Fluid Mech., 470 (2002), pp. 85-90.

[19] C. M. Linton And P. McIver, Embedded trapped modes in water waves and acoustics, Wave Motion, 45 (2007), pp. 16-29.

[20] C. M. Linton, R. Porter, And I. Thompson, Scattering by a semi-infinite periodic array and the excitation of surface waves, SIAM J. Appl. Math., 67 (2007), pp. 1233-1258.

[21] C. M. Linton And I. Thompson, One- and two-dimensional lattice sums for the threedimensional Helmholtz equation, J. Comput. Phys., 228 (2009), pp. 1815-1829.

[22] Z. Liu, C. T. Chan, P. Sheng, A. L. Goertzen, and J. H. Page, Elastic wave scattering by periodic structures of spherical objects: Theory and experiment, Phys. Rev. B, 62 (2000), pp. 2446-2457.

[23] P. A. Martin, Multiple Scattering. Interaction of Time-Harmonic Waves with N Obstacles, Cambridge University Press, Cambridge, UK, 2006.

[24] K. Maslov And V. K. KinRa, Acoustic response of a periodic layer of nearly rigid spherical inclusions in an elastic solid, J. Acoust. Soc. Amer., 106 (1999), pp. 3081-3088.

[25] K. Maslov, V. K. Kinra, and B. K. Henderson, Elastodynamic response of a coplanar periodic layer of elastic spherical inclusions, Mech. Mater., 32 (2000), pp. 785-795.

[26] P. McIver, C. M. Linton, ANd M. MCIver, Construction of trapped modes for wave guides and diffraction gratings, R. Soc. Lond. Proc. Ser. A Math. Phys. Eng. Sci., 454 (1998), pp. 2593-2616.

[27] A. Modinos, Scattering of electromagnetic waves by a plane of spheres-formalism, Phys. A, 141 (1987), pp. 575-588.

[28] R. Porter AND D. V. Evans, Rayleigh-Bloch surface waves along periodic gratings and their connection with trapped modes in waveguides, J. Fluid Mech., 386 (1999), pp. 233-258.

Copyright (c) by SIAM. Unauthorized reproduction of this article is prohibited. 
[29] R. Porter And D. V. Evans, Embedded Rayleigh-Bloch surface waves along periodic rectangular arrays, Wave Motion, 43 (2005), pp. 29-50.

[30] I. E. Psarobas, N. Stefanou, A. Modinos, and V. Yannopapas and, Scattering of elastic waves by periodic arrays of spherical bodies, Phys. Rev. B, 62 (2000), pp. 278-291.

[31] R. Sainidou, N. Stefanou, I. E. Psarobas, and A. Modinos, Scattering of elastic waves by a periodic monolayer of spheres, Phys. Rev. B, 66 (2002), article 024303.

[32] R. A. Shore and A. D. Yaghjian, Travelling electromagnetic waves on linear periodic arrays of lossless spheres, Electron. Lett., 41 (2005), pp. 578-579.

[33] R. A. Shore And A. D. YaghJian, Traveling waves on two- and three-dimensional periodic arrays of lossless scatterers, Radio Sci., 42 (2007), article RS6S21.

[34] N. Stefanou And A. Modinos, Scattering of light from a two-dimensional array of spherical particles on a substrate, J. Phys. Condens. Matter, 3 (1991), pp. 8135-8148.

[35] A. J. Stone And C. P. Wood, Root-rational-fraction package for exact calculation of vectorcoupling coefficients, Comput. Phys. Comm., 21 (1980), pp. 195-205.

[36] S. V. Sukhinin, The whispering surface effect, J. Appl. Math. Mech., 63 (1999), pp. 863-876.

[37] Y. TANAKa AND S.-I. TAmura, Surface acoustic waves in two-dimensional periodic elastic structures, Phys. Rev. B, 58 (1998), pp. 7958-7965.

[38] I. Thompson And C. M. Linton, On the excitation of a closely spaced array by a line source, IMA J. Appl. Math., 72 (2007), pp. 476-497.

[39] V. Twersky, Multiple scattering of sound by a periodic line of obstacles, J. Acoust. Soc. Amer., 53 (1973), pp. 96-112.

[40] V. Twersky, Lattice sums and scattering coefficients for the rectangular planar array, J. Math. Phys., 16 (1975), pp. 644-657.

[41] V. Twersky, Low frequency coupling in the planar rectangular lattice, J. Math. Phys., 16 (1975), pp. 658-666.

[42] V. Twersky, Multiple scattering of waves by the double periodic planar array of obstacles, J. Math. Phys., 16 (1975), pp. 633-643.

[43] J. Y. Vaishnav, J. D. Walls, M. Apratim, and E. J. Heller, Matter-wave scattering and guiding by atomic arrays, Phys. Rev. A, 76 (2007), article 013620.

[44] Y. Xu, Reflection and transmission of a plane wave by an array of cavities in the interface of two solids, J. Appl. Mech., 59 (1992), pp. 102-108.

Copyright $@$ by SIAM. Unauthorized reproduction of this article is prohibited. 